\title{
Corrigendum to "Haptoglobin Genotype and Outcome after Subarachnoid Haemorrhage: New Insights from a Meta-Analysis"
}

\author{
Ben Gaastra $\left(\mathbb{D},{ }^{1}\right.$ James Glazier, $^{2}$ Diederik Bulters, ${ }^{1}$ and Ian Galea ${ }^{2}$ \\ ${ }^{1}$ Wessex Neurological Centre, University Hospital Southampton, Southampton SO21 2AS, UK \\ ${ }^{2}$ Clinical Neurosciences, Clinical and Experimental Sciences, Faculty of Medicine, University of Southampton, \\ Southampton SO16 6YD, UK
}

Correspondence should be addressed to Ben Gaastra; bgaastra@nhs.net

Received 6 February 2018; Accepted 11 March 2018; Published 2 July 2018

Copyright (c) 2018 Ben Gaastra et al. This is an open access article distributed under the Creative Commons Attribution License, which permits unrestricted use, distribution, and reproduction in any medium, provided the original work is properly cited.

In the article titled "Haptoglobin Genotype and Outcome after Subarachnoid Haemorrhage: New Insights from a Meta-Analysis" [1], there were errors that have been corrected in the revised version shown below.

(i) The Results section has been updated.

(ii) Table 3 has been corrected.

(iii) Figures 2 and 3 have been corrected.

\begin{abstract}
Haptoglobin (Hp) is a plasma protein involved in clearing extracellular haemoglobin and regulating inflammation; it exists in two genetic variants ( $\mathrm{Hp} 1$ and $\mathrm{Hp} 2)$. In a metaanalysis of six published studies, we confirm that $\mathrm{Hp}$ genotype affects short-term outcome (cerebral vasospasm and/or delayed cerebral ischemia) after subarachnoid haemorrhage $(\mathrm{SAH})$ but not long-term outcome (Glasgow Outcome Score and modified Rankin Scale between one and three months). A closer examination of the heterozygous group revealed that the short-term outcome of Hp2-1 individuals clustered with that of Hp1-1 and not with that of Hp2-2, suggesting that the presence of one Hp1 allele was sufficient to confer protection. Since the presence of the $\mathrm{Hp}$ dimer is the only common feature between Hp11 and $\mathrm{Hp} 2-1$ individuals, the absence of this $\mathrm{Hp}$ moiety is most likely to underlie vasospasm in $\mathrm{Hp} 2-2$ individuals.
\end{abstract}

These results have implications for prognosis after $\mathrm{SAH}$ and will inform further research into $\mathrm{Hp}$-based mechanism of action and treatment.

\section{Introduction}

Haptoglobin (Hp) is an acute phase protein that binds to extracellular haemoglobin $(\mathrm{Hb})$ with very high affinity. The resulting $\mathrm{Hp}-\mathrm{Hb}$ complex is scavenged via CD163 expressed by cells of myeloid lineage [1]. There are two Hp alleles, Hp1 and $\mathrm{Hp} 2 . \mathrm{Hp} 2$ is a longer protein which arose during an intragenic duplication event affecting exons 3 and 4 in the Hpl gene. Recent data suggests that this happened at some point very early in human evolution, followed by recurring exonic deletions to reestablish modern $\mathrm{Hp1}$ [2]. Individuals can express one of three Hp genotypes: Hp1-1, Hp2-1, and $\mathrm{Hp} 2-2$. The alpha chain of $\mathrm{Hp}$ has one cysteine residue in $\mathrm{Hp} 1$ but two cysteine residues in $\mathrm{Hp} 2$. These cysteine residues can form intermolecular disulphide bonds to give rise to Hp molecules of different sizes [3]. Hp1-1 homozygotes only form $\mathrm{Hp}$ dimers and Hp2-2 homozygotes only form higher-order Hp polymers, while Hp2-1 heterozygous individuals form both $\mathrm{Hp}$ dimer and higher-order Hp polymers. In $\mathrm{Hp}$ 2-2 homozygotes, polymers are cyclic (i.e., $\operatorname{Hp}(\alpha 2 \beta) n$ where $n=3$ and above). In Hp2-1 heterozygotes, linear polymers form since polymer growth is arrested by Hp1 at both ends (i.e., $\operatorname{Hp}(\alpha 1 \beta) 2(\alpha 2 \beta) n$ where $n=0$ and above) [4].

Aneurysmal subarachnoid haemorrhage (aSAH) carries substantial morbidity and mortality. A common and serious complication of aSAH is that of cerebral vasospasm (CV). 
TABLE 1: Reported differences between Hp types, relevant to SAH.

\begin{tabular}{|c|c|c|}
\hline Function & No difference between Hp types & Difference between Hp types \\
\hline Hp expression & & $\begin{array}{l}\text { Serum Hp1-1 is higher than Hp2-2, with Hp2-1 } \\
\text { intermediate, in many populations tested, including } \\
\text { European (Belgian [37-39], Iceland [40]), East Asian } \\
\text { (Japanese [41], Koreans [42]), and African (Black } \\
\text { Zimbabweans [43], Gabonese [44], Papuans [45]). }\end{array}$ \\
\hline $\begin{array}{l}\text { Haemoglobin binding: } \\
\text { capacity per Hp monomer }\end{array}$ & $\begin{array}{l}\text { (1) Ultrafiltration assay of } \\
\text { uncomplexed Hb [46] } \\
\text { (2) Mass spectrometry [24] }\end{array}$ & \\
\hline Haemoglobin binding: affinity & $\begin{array}{l}\text { (1) Surface plasmon resonance [47] } \\
\text { (2) Surface plasmon resonance [24] } \\
\text { (3) Spectrophotometric signal of } \\
\text { Hp-Hp interaction [48] }\end{array}$ & \\
\hline $\begin{array}{l}\text { Inhibition of } \mathrm{Hb} \text {-mediated } \\
\text { oxidation }\end{array}$ & $\begin{array}{l}\text { (1) Reduction in low-density } \\
\text { lipoprotein oxidation [24] } \\
\text { (2) Reduction in Hb intrinsic redox } \\
\text { potential [48] } \\
\text { (3) Reduction in Hb autooxidation [17] }\end{array}$ & $\begin{array}{l}\text { (1) Hp1-1 is better than Hp2-2 at inhibiting protein } \\
\text { and lipid oxidation [49]. } \\
\text { (2) Hp1-1 is better than Hp2-2 at inhibiting oxidation } \\
\text { of linolenic acid and low-density lipoprotein [46]. } \\
\text { (3) Hp1-1 is better than Hp2-2 at inhibiting lipid } \\
\text { peroxidation [47]. }\end{array}$ \\
\hline Interaction with CD163: affinity & & $\begin{array}{l}\text { (1) } \mathrm{Hp} 2-2 \text { is better than } \mathrm{Hp} 1-1 \text {, by surface plasmon } \\
\text { resonance and binding of radioiodinated } \mathrm{Hp}-\mathrm{Hb} \\
\text { complexes in vitro [1]. } \\
\text { (2) } \mathrm{Hp} 2-2 \text { is better than } \mathrm{Hp} 1-1 \text {, by binding of } \\
\text { radioiodinated } \mathrm{Hp}-\mathrm{Hb} \text { complexes in vitro [50]. }\end{array}$ \\
\hline $\begin{array}{l}\text { Interaction with CD163: } \\
\text { uptake of } \mathrm{Hp}-\mathrm{Hb} \text { complexes }\end{array}$ & $\begin{array}{l}\text { Plasma half-life of } \mathrm{Hp}-\mathrm{Hb} \text { complexes } \\
\text { after injection in guinea pigs [24] }\end{array}$ & $\begin{array}{l}\text { (1) } \mathrm{Hp} 2-2 \text { is better than } \mathrm{Hp} 1-1 \text {, by measurement } \\
\text { of free } \mathrm{Hb} \text { in humans [51]. } \\
\text { (2) } \mathrm{Hp} 1-1 \text { is better than } \mathrm{Hp} 2-2 \text {, by uptake of } \\
\text { radioiodinated } \mathrm{Hp}-\mathrm{Hb} \text { complexes in human } \\
\text { cells in vitro [50]. }\end{array}$ \\
\hline Effects on inflammation & & $\begin{array}{l}\text { Binding of } \mathrm{Hp} 1-1-\mathrm{Hb} \text { complexes to } \mathrm{CD} 163 \text { results } \\
\text { in secretion of the anti-inflammatory cytokine } \\
\text { IL-10 }[19,20] \text {. }\end{array}$ \\
\hline
\end{tabular}

Prolonged or pronounced vasoconstriction of major cerebral blood vessels can lead to delayed cerebral ischemia (DCI), which occurs in up to $30 \%$ of individuals who survive aSAH, manifesting as new focal neurological signs and/or deterioration in level of consciousness. Together, CV and DCI contribute to short-term outcome, by increasing short-term morbidity, hospital stay, and costs [5]. Longer-term outcome and functional status after aSAH are typically assessed using the modified Rankin Scale (mRS) or the Glasgow Outcome Scale (GOS).

$\mathrm{Hp}$ alleles profoundly affect outcome after intracranial haemorrhage, such as aSAH; $\mathrm{Hp} 2$ confers a poorer prognosis, with odds ratios of up to 4 being reported [6]. The underlying mechanism remains to be established. There are three potential biological mechanisms to explain this phenomenon: difference in $\mathrm{Hp}$ expression, $\mathrm{Hp}$ function, and $\mathrm{Hp}-\mathrm{Hb}$ complex size. There is agreement that serum $\mathrm{Hp}$ expression is highest in Hp1-1 individuals, intermediate in Hp2-1, and lowest in Hp2-2 individuals (Table 1). With respect to functional aspects of $\mathrm{Hp}$ relevant to $\mathrm{SAH}$, these include affinity of $\mathrm{Hp}$ binding to $\mathrm{Hb}, \mathrm{Hb}$ binding capacity of $\mathrm{Hp}$, protection from Hb's redox toxicity, affinity to CD163, CD163mediated uptake, and effects on inflammation (Table 1). There is a lack of agreement as to whether Hp1 and Hp2 differ with respect to some of these aspects, and in which direction, as reviewed in Table 1 . The third potential mechanism relates to the fact that the dimer produced by $\mathrm{Hp} 1-1$ and Hp2-1 individuals is smaller than other higher-order polymers produced by Hp2-2 individuals. This may be important since solute drainage from the brain along the glymphatic pathway has a size selectivity [7]. Drainage of $\mathrm{Hp}-\mathrm{Hb}$ complexes from the brain to the circulation may be important since CD163 binding sites are reduced and saturated in the brain $[8,9]$.

The outcome of Hp2-1 individuals, when compared to that of Hp1-1 and Hp2-2, is likely to shed light on the mechanism underlying the prognostic effect of $\mathrm{Hp}$. Differences in function between $\mathrm{Hp}$ types are likely to result in a dosedependent effect between genotypes, while a predominant effect of the Hp dimer is likely to result in similar outcomes in Hp2-1 and Hp1-1 individuals, but different from Hp2-2 individuals. So far, small sample sizes have precluded meaningful comparison of the outcome of the heterozygous versus homozygous genotypes. We hypothesised that Hp2-2 individuals are at greater risk of poor short- and long-term outcomes after aSAH. A meta-analysis of published studies was performed with the following objectives: (1) to confirm the effect of Hp genotype on outcome after aSAH and (2) 


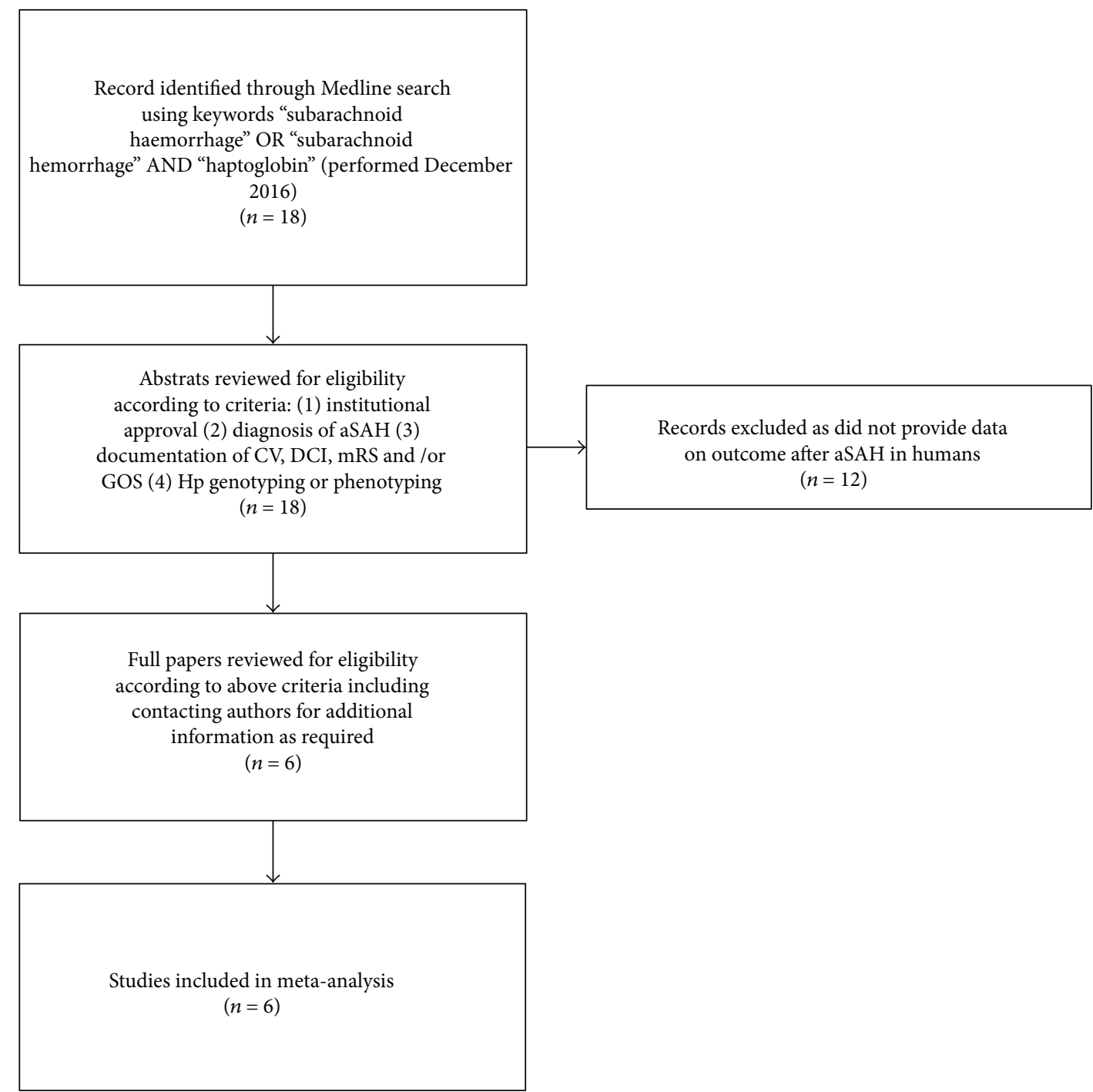

FIGURE 1: Flow diagram of studies selected for inclusion. If additional information was required, the authors were contacted by email.

to compare the outcome of Hp2-1 individuals with that of Hp1-1 and Hp2-2 individuals and so provide mechanistic insight. In summary, an unfavourable effect of the Hp2-2 genotype on short-term outcome was confirmed. The outcome of Hp2-1 individuals clustered with that of Hp1-1, suggesting that the presence of one $\mathrm{Hp} 1$ allele was sufficient to confer protection. Mechanistically, this is in keeping with the hypothesis that the Hp dimer is essential, possibly due to its small size.

\section{Materials and Methods}

Meta-analysis was conducted in accordance with the PRISMA [10] (Supplementary Table 1 available online at https://doi.org/10.1155/2017/6747940) and Cochrane Collaboration guidelines. Data from six studies were included in the meta-analysis (see Figure 1 for search criteria and Table 2 for summary of studies included; all studies were observational) $[6,8,11-14]$. For each individual study, bias was assessed using the Newcastle-Ottawa Scale for quality assessment of nonrandomised studies [15]. This assessment is based on 3 domains (selection, comparability, and outcome) and allows a study to be scored between 0 and 8 . Authors BG, DB, and IG independently scored each study; if there was disagreement between scores, an average was taken. All studies included in this analysis scored 5 and were therefore considered to be at low risk of bias (Supplementary Table 2). Although all studies were assessed to be at low risk of bias, the scoring system highlighted the inclusion of higher Fisher grade patients, limited intrastudy controls, and short duration of follow-up as potential sources of bias. Tests for funnel plot asymmetry were not performed as the meta-analysis only included 6 studies, in keeping with recommendations from the Cochrane Collaboration. Shortand long-term outcomes were derived from the six studies and analysed separately. Short-term outcome was defined as $\mathrm{CV}$ and/or DCI during the inpatient period, as determined by any means, including cerebral angiography, transcranial Doppler ultrasonography, and clinical or radiological evidence of DCI. If both CV and DCI values were available, then 


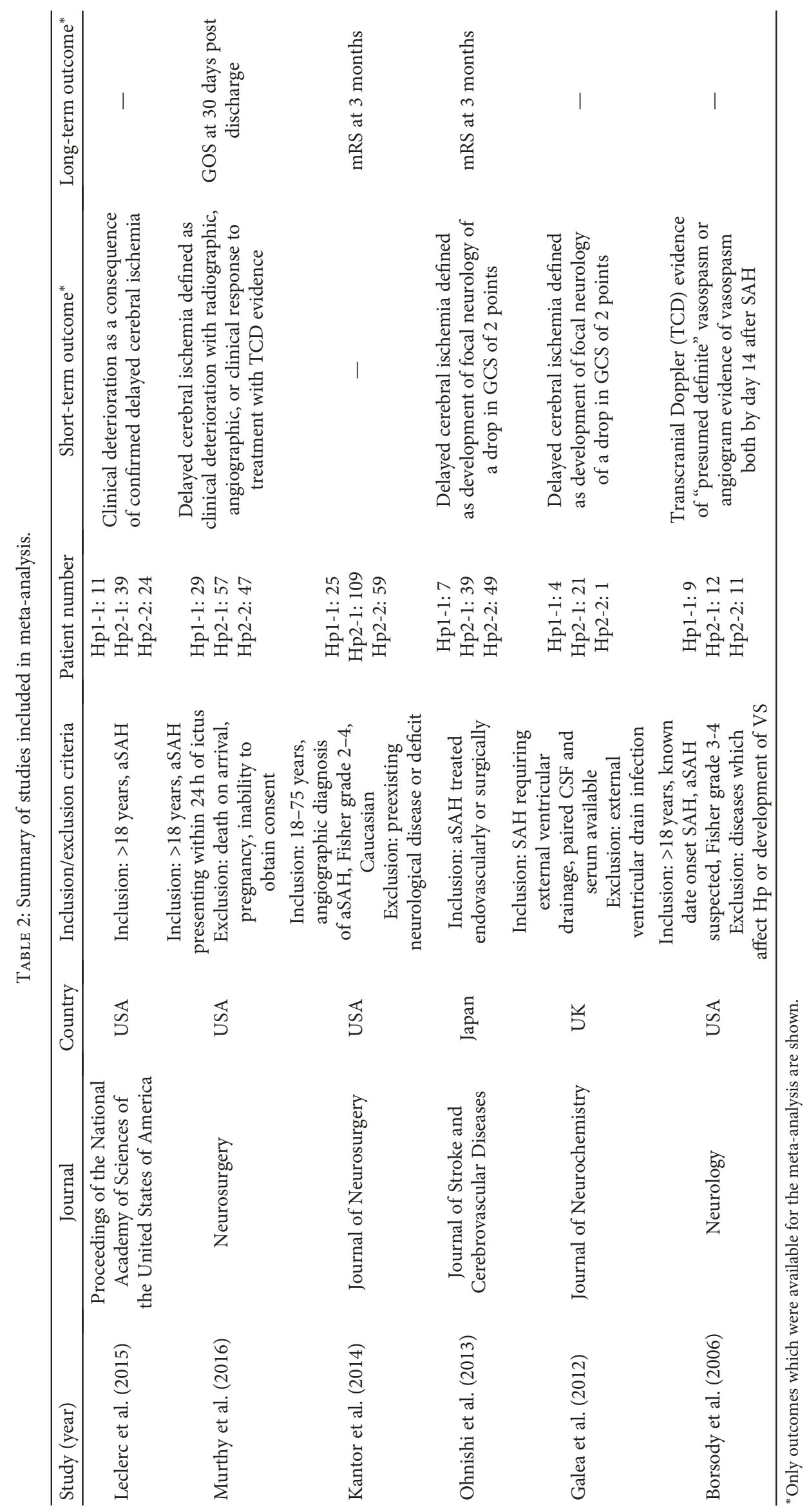


TABLE 3: Short and long-term outcome after aSAH.

\begin{tabular}{|c|c|c|c|c|c|c|c|c|}
\hline \multirow{2}{*}{$\begin{array}{l}\text { Comparison groups } \\
\text { Group A }\end{array}$} & \multirow[b]{2}{*}{ Group B } & \multirow{2}{*}{$\begin{array}{c}N \text { total } \\
\text { Group } \mathrm{A}+\mathrm{B}\end{array}$} & \multirow{2}{*}{$\begin{array}{l}\text { Odds ratio for poor outcome } \\
\text { Group A/B }\end{array}$} & \multirow{2}{*}{$\mathrm{Z}$} & \multirow[b]{2}{*}{$p$} & \multicolumn{3}{|c|}{ Heterogeneity } \\
\hline & & & & & & $\mathrm{Chi}^{2}$ & Df & $I^{2}$ \\
\hline \multicolumn{9}{|l|}{ Short-term outcome } \\
\hline $\mathrm{Hp} 2-2$ & Hp1-1 & $192(132+60)$ & $2.37(1.12,5.04)$ & 2.26 & $0.02^{*}$ & 3.93 & 3 & $24 \%$ \\
\hline Hp2-1 & Hp1-1 & $228(168+60)$ & $1.53(0.74,3.14)$ & 1.16 & 0.25 & 5.92 & 4 & $32 \%$ \\
\hline Hp2-2 & Нp2-1 & $300(132+168)$ & $1.90(1.11,3.25)$ & 2.34 & $0.02^{*}$ & 1.79 & 4 & $0 \%$ \\
\hline $\mathrm{Hp} 2-2$ & Hp1-1 \& Hp2-1 & $360(132+228)$ & $2.07(1.26,3.41)$ & 2.87 & $0.004^{*}$ & 1.77 & 4 & $0 \%$ \\
\hline Hp2-1 \& Hp2-2 & Hp1-1 & $360(300+60)$ & $1.96(0.99,3.86)$ & 1.94 & 0.05 & 5.11 & 4 & $22 \%$ \\
\hline \multicolumn{9}{|l|}{ Long-term outcome } \\
\hline Hp2-2 & Hp1-1 & $216(155+61)$ & $1.61(0.81,3.16)$ & 1.37 & 0.17 & 4.96 & 2 & $60 \%$ \\
\hline Hp2-1 & Hp1-1 & $266(205+61)$ & $1.28(0.65,2.51)$ & 0.72 & 0.47 & 3.58 & 2 & $44 \%$ \\
\hline Hp2-2 & Нp2-1 & $360(155+205)$ & $1.34(0.85,2.10)$ & 1.27 & 0.20 & 0.55 & 2 & $0 \%$ \\
\hline $\mathrm{Hp} 2-2$ & Hp1-1 \& Hp2-1 & $421(155+266)$ & $1.37(0.89,2.10)$ & 1.44 & 0.15 & 1.55 & 2 & $0 \%$ \\
\hline Hp2-1 \& Hp2-2 & Hp1-1 & $421(360+61)$ & $1.41(0.75,2.65)$ & 1.06 & 0.29 & 4.26 & 2 & $53 \%$ \\
\hline
\end{tabular}

${ }^{*} p<0.05 ; Z$ : test for overall effect.

DCI data was used in preference due to greater clinical relevance. Both DCI and CV are features occurring in the initial period after $\mathrm{SAH}$, which have a well-demonstrated impact on short-term morbidity, inpatient stay duration, and economic costs, justifying their joint qualification as short-term outcome. Data for short-term outcome was available from five studies (Borsody et al. [11], Galea et al. [8], Ohnishi et al. [14], Leclerc et al. [12], and Murthy et al. [13]) and was classified as either present or absent. Longterm outcome was defined as dichotomized $\mathrm{mRS}$ or GOS, between one and three months after aSAH. mRS and GOS scores of $0-2$ and 4-5, respectively, were considered as favourable outcome, with the rest of the scores being unfavourable. Data for long-term outcome was available from three studies at one month (Murthy et al. [13]) or three months (Ohnishi et al. [14], Kantor et al. [6]). Metaanalysis was conducted in Review Manager (RevMan) v5.3.3. The Mantel-Haenszel (M-H) method for calculating the weighted pooled odds ratio in a fixed effects model was used. Significance was accepted to be present at $p<0.05$.

\section{Results}

553 aSAH patients were included; short-term and long-term outcome data were available for 360 and 421 patients, respectively. Results are presented in Table 3; forest plots are presented in Figures 2 and 3. The Hp2-2 genotype imparted a worse short-term prognosis compared to Hp1-1 ( $\mathrm{OR}=2.37$, $95 \% \mathrm{CI}=1.12-5.04, p=0.02)$. The significance of this relationship was increased by including Hp2-1 with Hp1-1 cases $(\mathrm{OR}=2.07,95 \% \mathrm{CI}=1.26-3.41, p=0.004)$ and decreased by including $\mathrm{Hp} 2-1$ with $\mathrm{Hp} 2-2$ cases $(\mathrm{OR}=1.96,95 \%$ $\mathrm{CI}=0.99-3.86, p=0.05)$, suggesting that the outcome of Hp2-1 patients more closely resembled that of Hp1-1 patients. In support of this explanation, the short-term outcome of Hp2-1 patients was significantly different from that of $\mathrm{Hp} 2-2$ patients $(\mathrm{OR}=1.90,95 \% \mathrm{CI}=1.11-3.25, p=0.02)$, but not that of $\mathrm{Hp} 1-1$ patients $(\mathrm{OR}=1.53,95 \% \mathrm{CI}=0.74-$
$3.14, p=0.25)$. No effect of Hp genotype on long-term outcome was observed.

\section{Discussion}

$\mathrm{Hp}$ can protect against $\mathrm{Hb}$ toxicity in a number of ways. First, $\mathrm{Hp}$ lowers the redox potential of $\mathrm{Hb}$ by binding it. This is achieved by stabilizing ferryl iron [16] and globinbased amino acid radicals $[16,17]$, sites within or close to the interface between $\mathrm{Hp}$ and $\mathrm{Hb}$ [18], preventing these reactive entities from participating in redox reactions. Second, $\mathrm{Hp}$ targets $\mathrm{Hb}$ for degradation, since $\mathrm{Hb}-\mathrm{Hp}$ is recognized and cleared by CD163 [1]. Third, Hp induces an anti-inflammatory response (e.g., interleukin-10 secretion $[19,20])$, which serves to balance $\mathrm{Hb}$ or heme-induced proinflammatory effects (e.g., tumour necrosis factor [21, 22] and interleukin-1 [23] secretion). There is controversy as to whether some of these functions differ between $\mathrm{Hp}$ types (Table 1). It is important to note that $\mathrm{Hp}$ binding to $\mathrm{Hb}$ does not affect its capacity to scavenge nitric oxide $[24,25]$. Hence, any nitric oxide-mediated mechanistic basis for differences in vasospasm between $\mathrm{Hp}$ genotypes is likely linked to clearance of $\mathrm{Hb}$.

A study of experimental SAH in mice clearly demonstrated differences between $\mathrm{Hp} 1$ and Hp2 [26]. Mice only express Hp1, but mice genetically engineered to express $\mathrm{Hp} 2$ in place of Hp1 had a poorer outcome after SAH, compared to Hp1 wild-type mice [26]. However, this study did not examine Hp2-1 mice. This meta-analysis has confirmed that the Hp2-2 genotype confers a worse short-term outcome versus the Hp1-1 genotype in humans. Moreover, the shortterm outcome of $\mathrm{Hp} 2-1$ patients clusters with that of $\mathrm{Hp} 1-1$ patients, suggesting that the presence of one $\mathrm{Hp} 1$ allele is sufficient to confer protection over $\mathrm{Hp} 2$.

The findings in the Hp2-1 heterozygous individuals have mechanistic implications. Functional mechanisms such as lowering $\mathrm{Hb}$ redox potential, CD163 uptake, or antiinflammatory effects would be expected to result in dosedependent differences in outcome between the three 


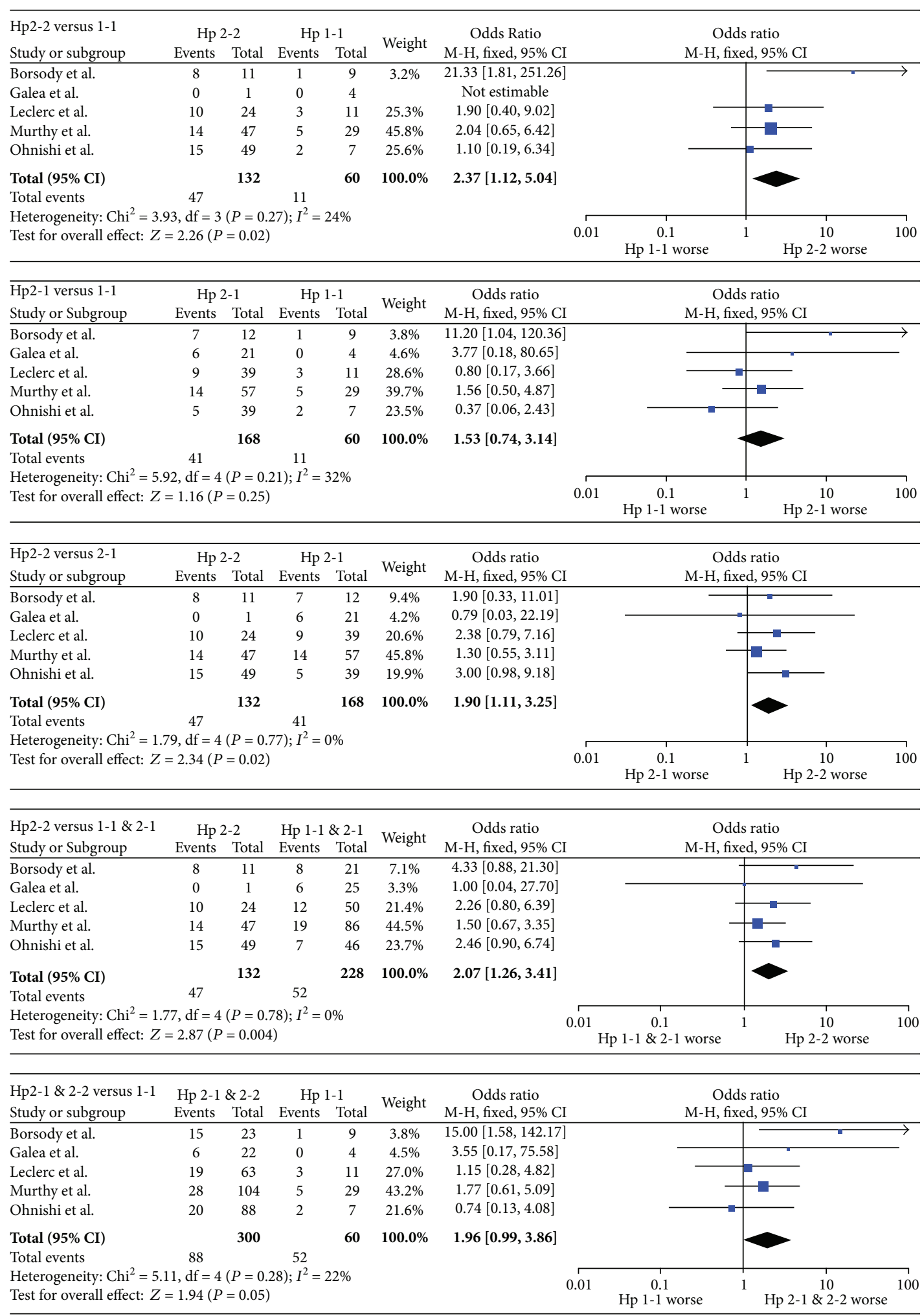

FIGURE 2: Forest plots for short-term outcome data. Short-term outcome was defined as CV and/or DCI during the inpatient period, as determined by any means, including cerebral angiography, transcranial Doppler ultrasonography, and clinical or radiological evidence of DCI.

genotypes. However, the short-term outcome of Hp2-1 was similar to that of Hp1-1. The common feature amongst Hp1-1 and Hp2-1 individuals is the presence of the Hp dimer, which therefore appears to be important in conferring protection. It is possible that the small size of the dimer facilitates drainage of the $\mathrm{Hp}-\mathrm{Hb}$ complexes from the brain. 


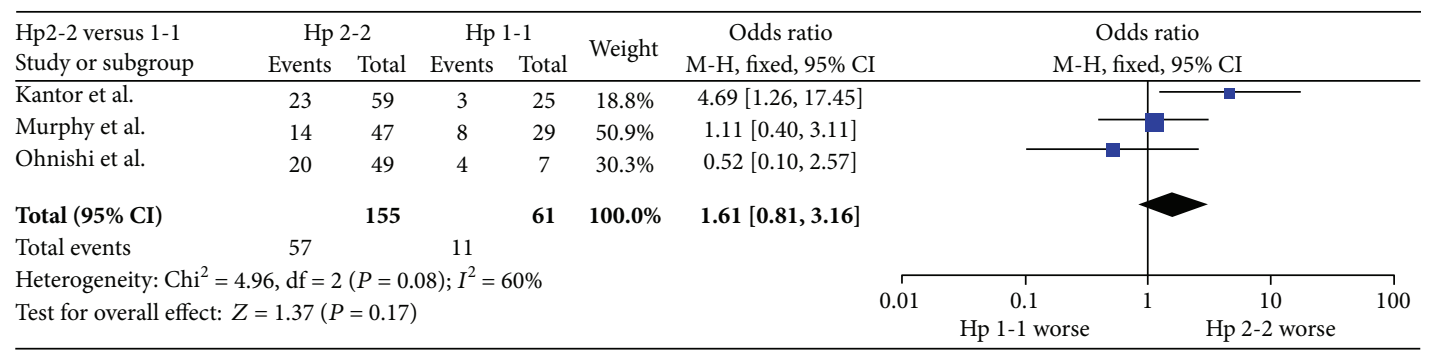

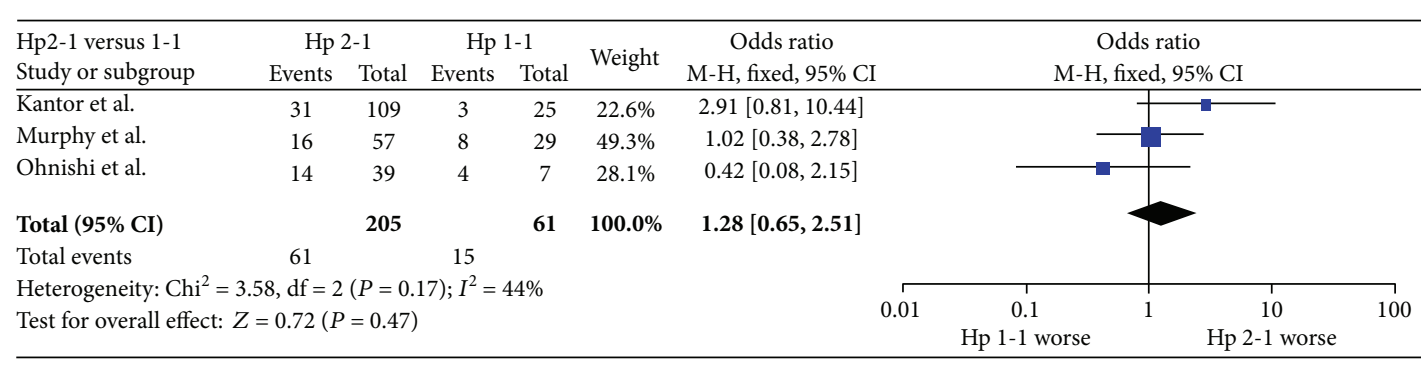

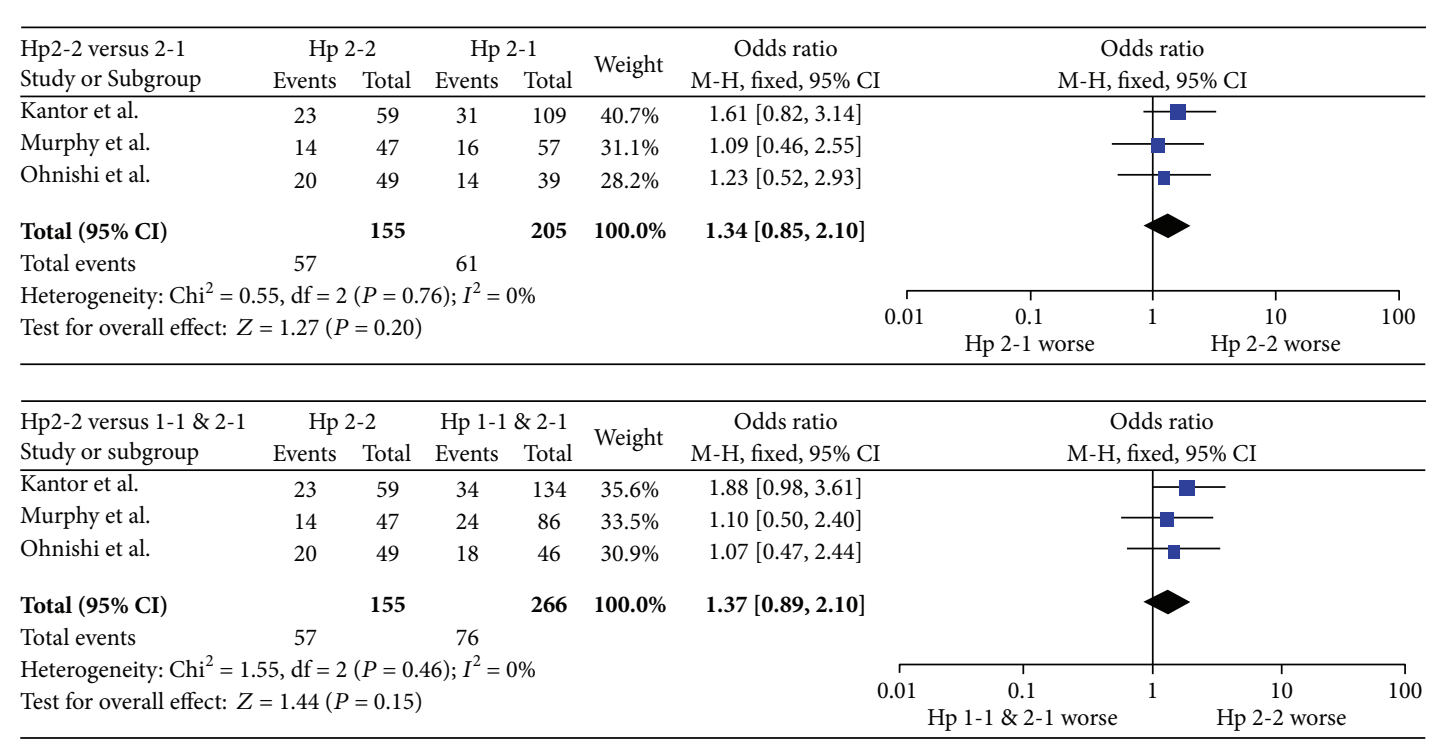

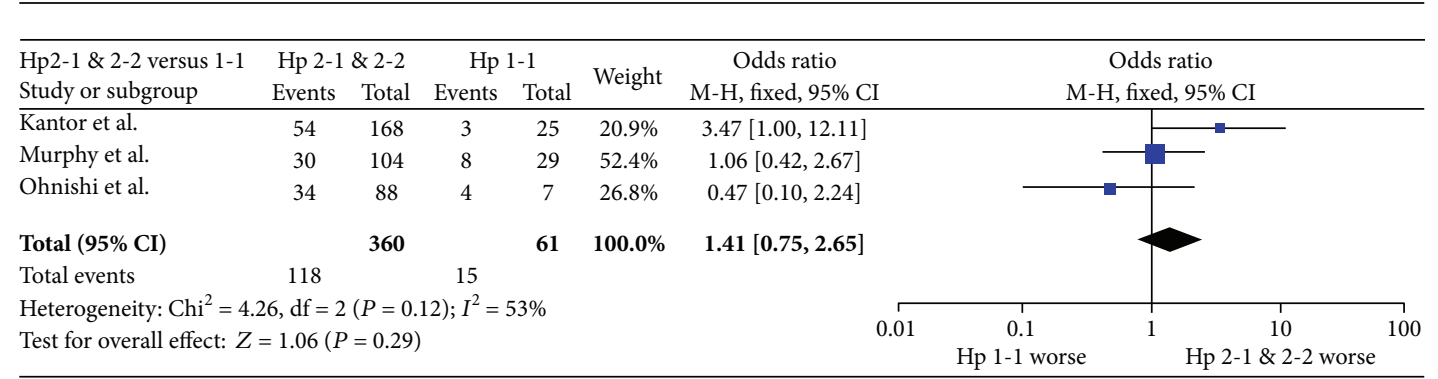

FIGURE 3: Forest plots for long-term outcome data. Long-term outcome was defined as dichotomized mRS or GOS between one and three months after aSAH.

Although several studies have shown upregulation of CD163 after intracerebral haemorrhage [27-30], CD163 binding sites are limiting after $\mathrm{SAH}$ since free $\mathrm{Hp}-\mathrm{Hb}$ complexes persist in the cerebrospinal fluid $[8,9]$, possibly compounded by soluble CD163 shedding [8]. For this reason, drainage of Hp$\mathrm{Hb}$ complexes out of the brain via the glymphatic pathway [7] may be important. There is evidence for a size selectivity in the glymphatic pathway [7] so that molecules with a molecular weight above $200 \mathrm{kDa}$ have reduced clearance.
The size of the Hp dimer in complex with $\mathrm{Hb}$ would be below this threshold $(180 \mathrm{kDa})$, while $\mathrm{Hb}$ in complex with $\mathrm{Hp}$ polymers of increasing valency would have higher molecular weights. The small size of the Hp dimer also enables it to enter the brain across the blood-brain barrier while higherorder polymers find it more difficult [31]. Hence, amongst all the Hp forms, the Hp dimer would be able to recycle into and out of the brain with greatest ease, clearing $\mathrm{Hb}$ from the brain in the process. In keeping with this explanation, a 
decrease in serum Hp occurs after aSAH, most marked in individuals with the highest blood-brain barrier disruption [8]. These speculations need to be addressed by experimental work to prove that $\mathrm{Hp} 1-1-\mathrm{Hb}$ complex size impacts on outcome by altering drainage of $\mathrm{Hb}$ out of the brain. It is still possible that $\mathrm{Hp} 1 / \mathrm{Hp} 2$ differences in lowering $\mathrm{Hb}$ redox potential, CD163-mediated $\mathrm{Hp}-\mathrm{Hb}$ uptake, or antiinflammatory action could affect outcome in a manner which is not dose dependent.

The findings of this meta-analysis are important for prognostication in the clinical setting, since the Hp2-2 status clearly reflects a group of individuals who may benefit from closer monitoring within a specialist neurointensive care unit. Hp genotype did not affect long-term outcome in this meta-analysis, despite a clear relationship with short-term outcome. This may be due to several reasons. CV may not be related to long-term outcome and this remains controversial [32]. Due to their relatively crude nature, the GOS and mRS scales may not be sufficiently sensitive to detect differences. Recently, two groups have demonstrated upregulation of neuronal CD163 expression after intracranial haemorrhage in nonhuman models $[30,33,34]$-if this finding is confirmed in humans, neurons in $\mathrm{Hp} 1$ individuals may accumulate more intracellular heme/iron, which is toxic [35]. It is possible that the short-term beneficial effects of Hp1-1 on vasospasm are balanced by the long-term deleterious effects of Hp1-1 on neuronal iron accumulation, so that long-term outcome is unaffected overall.

This study has a number of limitations. Long-term outcome combined one- and three-month outcomes; however, a sensitivity analysis excluding the one-month study did not change the finding that Hp genotype did not affect the long-term outcome. Short-term outcome was defined as $\mathrm{CV}$ and/or DCI, and these phenomena might not necessarily be equivalent [36]; however, a sensitivity analysis excluding CV showed that DCI-only outcome of Hp2-1 patients more closely resembled that of Hp1-1 patients: Hp1-1 and Hp2-1 versus Hp2-2 $(p=002$, OR $(\mathrm{CI})=1.9 \quad(1.12-3.22))$ and Hp1-1 versus Hp2-1 and Hp2-2 $(p=0$ 83, OR $(\mathrm{CI})=1.08$ (0.55-2.14)). We also noted that the majority of participants across all studies had high Fisher grade aSAH, so generalizability of the findings here to other patients with aSAH needs to be approached with caution. Although there was no evidence of significant heterogeneity (Table 3), prognostic factors may still have been distributed asymmetrically amongst studies and/or genotype groups; therefore, an individual patient level data analysis is warranted to weigh up Hp genotype against other prognostic covariates, in determining outcome after aSAH.

\section{Conclusions}

In conclusion, this study confirms the unfavourable effect of the $\mathrm{Hp} 2$ allele on short-term outcome after aSAH. It advances the field by showing that the presence of one Hp1 allele is sufficient to counter the unfavourable effect of $\mathrm{Hp} 2$. This suggests that the Hp dimer is the structural determinant of the association of the $\mathrm{Hp}$ polymorphism with outcome after SAH. Therapies aimed at augmenting Hp may work best if designed to mainly deliver $\mathrm{Hp} 1$, rather than elevate $\mathrm{Hp}$ nonspecifically, in $\mathrm{Hp} 2-1$ and Hp2-2 individuals. Experimental studies are needed to prove this hypothesis.

\section{Conflicts of Interest}

The authors declare that there is no conflict of interest regarding the publication of this article.

\section{Acknowledgments}

The authors thank Sheila Alexander, University of Pittsburgh.

\section{References}

[1] B. Gaastra, J. Glazier, D. Bulters, and I. Galea, "Haptoglobin genotype and outcome after subarachnoid haemorrhage: new insights from a meta-analysis," Oxidative Medicine and Cellular Longevity, vol. 2017, Article ID 6747940, 9 pages, 2017. 


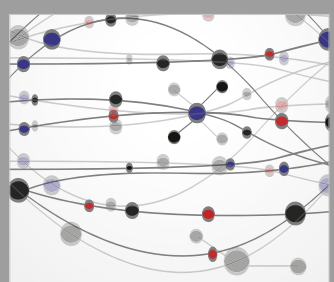

The Scientific World Journal
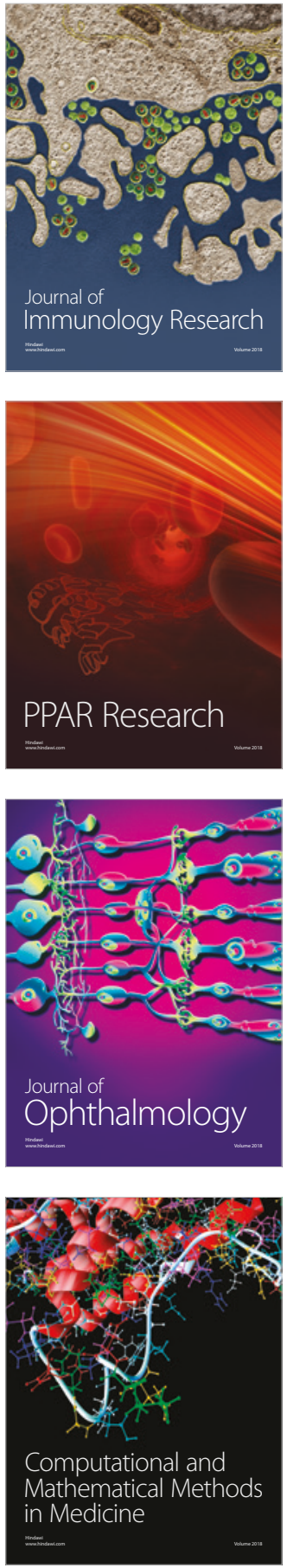

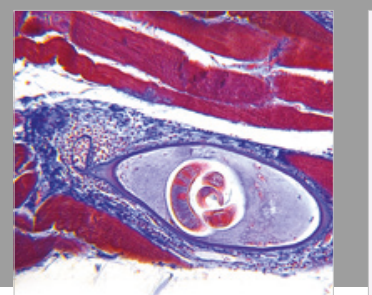

Gastroenterology Research and Practice

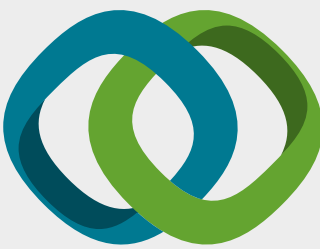

\section{Hindawi}

Submit your manuscripts at

www.hindawi.com
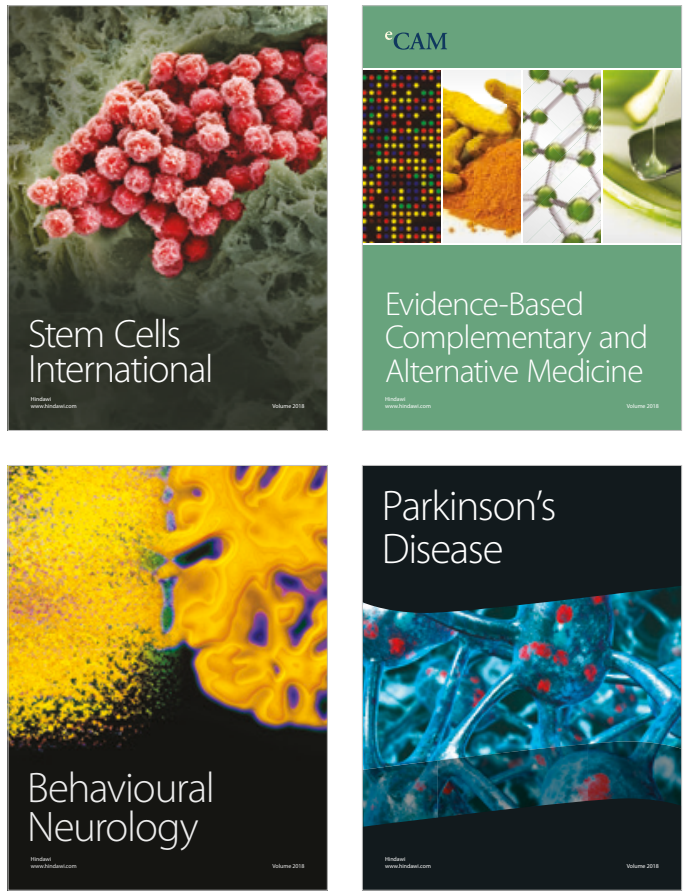

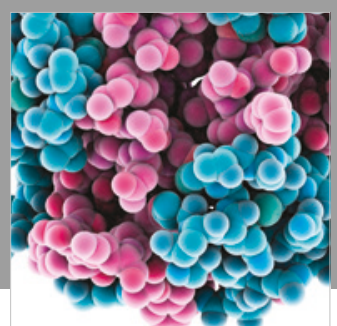

ournal of

Diabetes Research

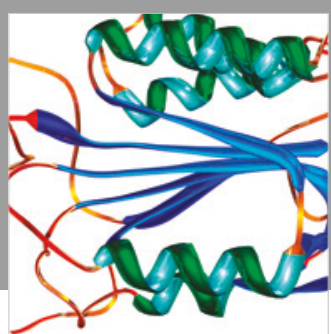

Disease Markers
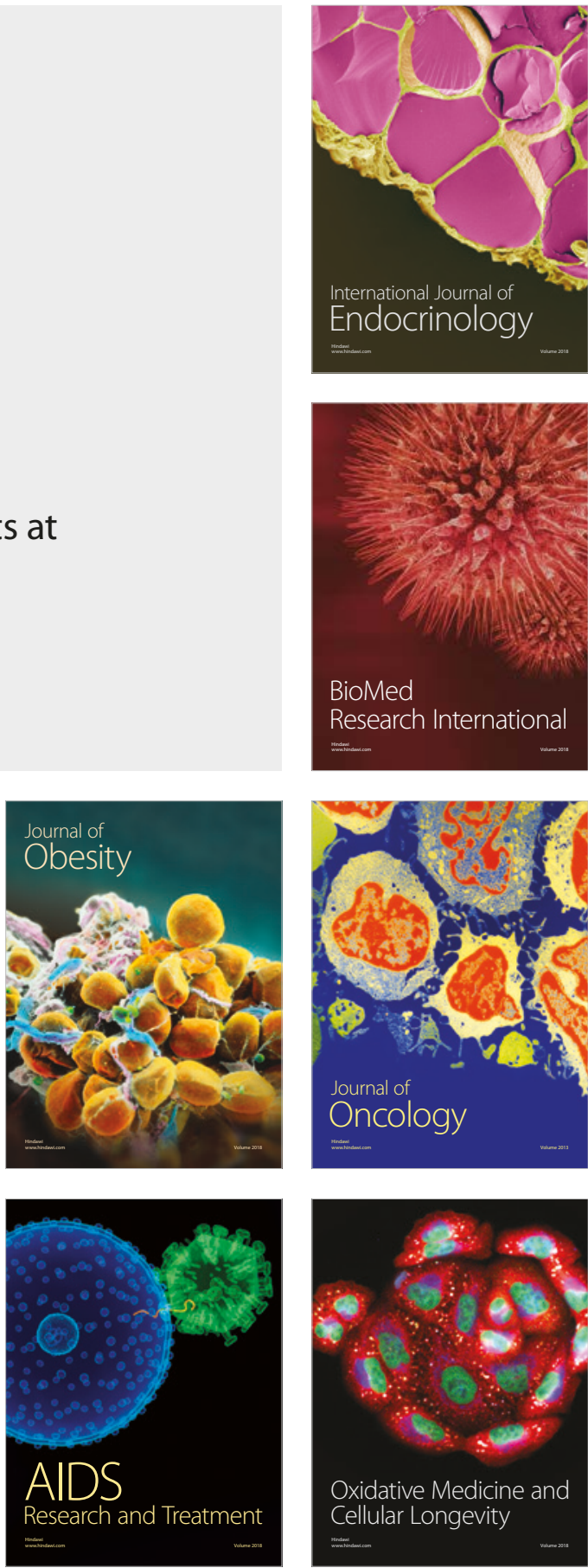